\title{
18 CM HIGH RESOLUTION OBSERVATIONS OF 15 EXTRAGALACTIC RADIO SOURCES
}

\author{
J. A. Waak, J. H. Spencer, R. S. Simon, and K. J. Johnston \\ E. O. Hulburt Center for Space Research \\ Naval Research Laboratory, Washington, D.C. 20375-5000
}

On 8-12 October 1981 a 5-station MkII VLBI experiment at $1660 \mathrm{MHz}$ was performed using antennas at Westford, Maryland Point, Green Bank, Fort Davis, and Owens Valley. Fif teen extragalactic sources (Table 1) were observed with an average synthesized beam of roughly 10 mas. The data were processed using the NRAO correlator, globally fringe-fit, and mapped with the AIPS package. In addition to the usual self-calibration techiques, corrections were made to eliminate baseline-dependent calibration errors. Some of the sources, for example $1641+399$ (3C345), display extended components not before observed at the dynamic range of the maps, which on the average is roughly 200:1.

The contour levels in the maps are normally at $+/-0.5,1,2,5,10,20,30,50$, 70 , and $90 \%$ of the peak flux. The actual lowest contour level and other map parameters for each source are given in Table 1. One negative contour is drawn for each source at the level corresponding to the lowest positive contour for that source.

TABLE 1: MAP PARAMETERS

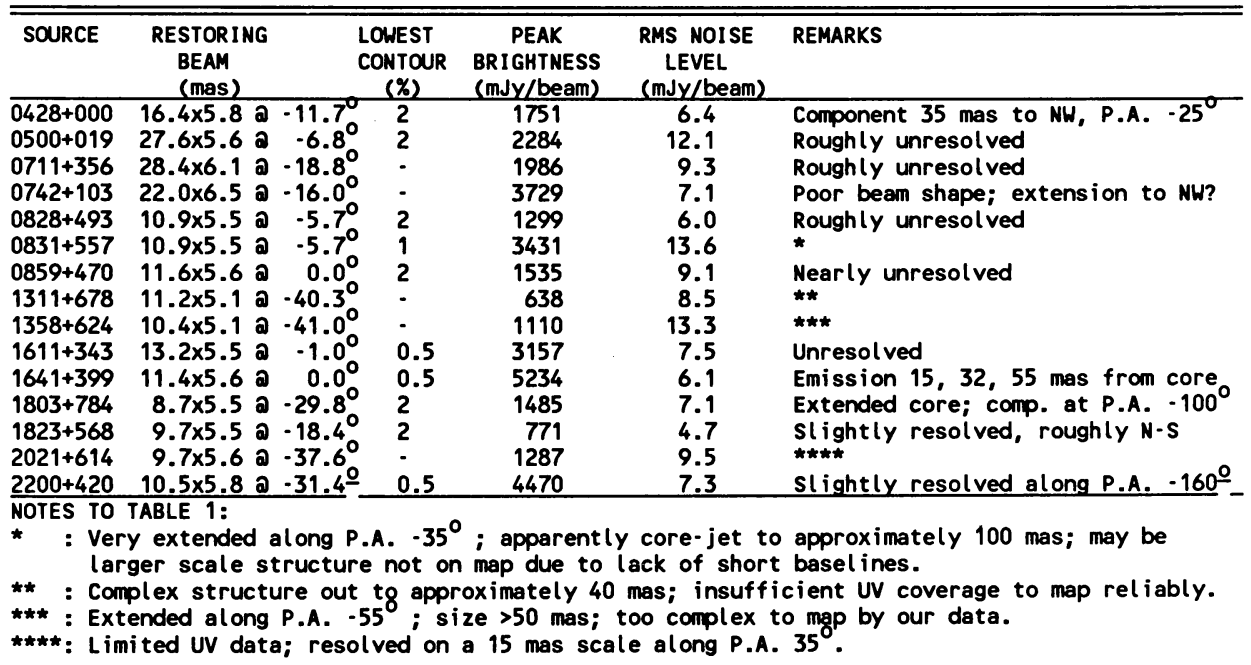

M. J. Reid and J. M. Moran (eds.), The Impact of VLBI on Astrophysics and Geophysics, 141-142.

(C) 1988 by the IAU. 


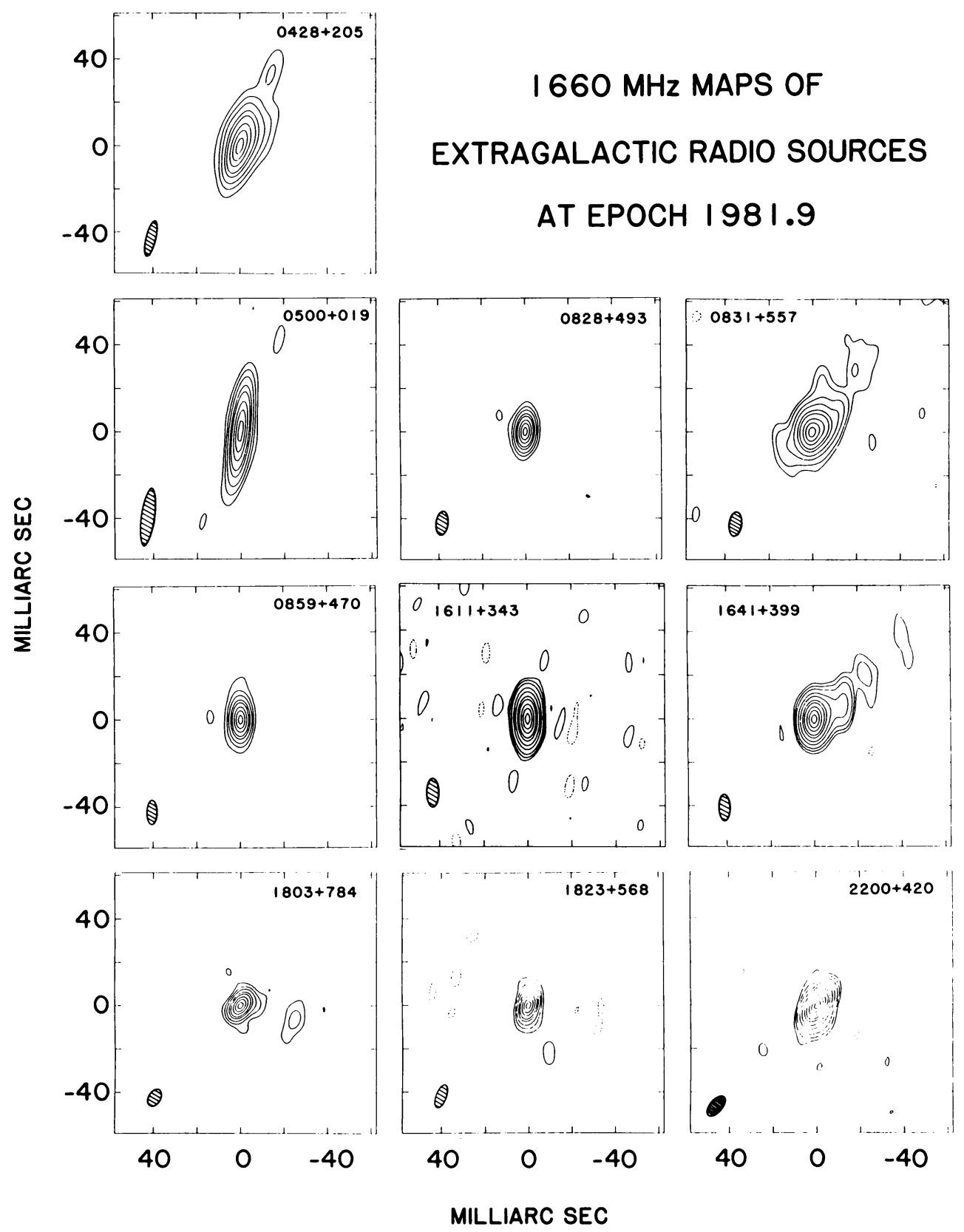

